\title{
La gestion de la crise sanitaire : une « expérimentalisation » du monde?
}

Health crisis management: an "experiment" of the world?

Alain Loute

\section{OpenEdition}

Journals

Édition électronique

URL : https://journals.openedition.org/grm/3294

DOI : $10.4000 / \mathrm{grm} .3294$

ISSN : 1775-3902

Éditeur

Groupe de Recherches Matérialistes

Référence électronique

Alain Loute, "La gestion de la crise sanitaire : une « expérimentalisation » du monde ? », Cahiers du GRM [En ligne], 18 | 2021, mis en ligne le 28 septembre 2021, consulté le 01 octobre 2021. URL : http:// journals.openedition.org/grm/3294; DOI : https://doi.org/10.4000/grm.3294

Ce document a été généré automatiquement le 1 octobre 2021.

(C) GRM - Association 


\title{
La gestion de la crise sanitaire : une " expérimentalisation » du monde?
}

\author{
Health crisis management: an "experiment" of the world?
}

\author{
Alain Loute
}

1 Les effets du Covid-19 sont multiples. S'ils se donnent avant tout à voir sur le plan sanitaire, ils sont aussi économiques, sociaux et politiques. Parmi les nombreuses analyses qui ont été produites à ce sujet, plusieurs textes pointent les risques que la crise ou plutôt sa gestion ont fait courir à la démocratie. À titre d'exemple, AnneEmmanuelle Bourgaux relève qu'à partir de mars 2020, en deux mois, une centaine d'arrêtés de pouvoirs spéciaux ont étê adoptés en Belgique :

Ce recours addictif aux pouvoirs spéciaux évince nos élues qui, seules, sont représentatif.ves de nos voix dans leur ensemble. Il entraine aussi un problème de contrôle : qui, parmi les citoyen'nes, la presse, les corps intermédiaires, réussit encore à suivre ? ${ }^{1}$

2 Pour le philosophe Marc Maesschalck ${ }^{2}$, la gestion de la crise sanitaire menée aura restreint l'ordre politique à sa forme la plus contestée intellectuellement et socialement depuis quarante ans, à savoir la structure d'une démocratie de représentation et de surveillance. Le confinement n'aura pas seulement restreint des libertés individuelles, il s'est traduit par la mise sous tutelle de l'espace public, ce dernier n'existant plus que sous une forme virtualisée. Face à la sphère politique professionnelle, appuyée dans sa gestion de la crise par des experts, la société civile s'est trouvée sans médiation, relais et porte-parole. Des associations de patients (Renaloo, la Ligue contre le cancer, de même que France Assos Santé), auditionnées par une commission sénatoriale en France, confirment ces diagnostics. Elles rapportent un réel «recul de la démocratie sanitaire »: «Médiatiquement et politiquement, nous étions en réaction plus qu'en construction d'une politique de santé $»^{3}$.

3 À mes yeux, un des éléments centraux pour comprendre cette mise en crise de la démocratie concerne la question de l'expertise. Bernadette Bensaude-Vincent nous invite précisément à lire la gestion de cette crise sanitaire depuis les rapports entre 
savoir et pouvoir. Pour elle, dans la gestion de la crise, le public a été réduit au silence, sommé d'obéir, pour son bien, aux injonctions du pouvoir et des experts :

Cette attitude infantilisante rappelle celle qui prévalut au XXème siècle quand philosophes et savants ne voyaient qu'un fossé entre savants et ignorants, un fossé grandissant à mesure des progrès de la science, condamnant « le profane » à vivre sous tutelle.

Barbara Stiegler a soutenu également que les modalités du gouvernement de la pandémie en France laissaient transparaître « la conviction du caractère irrationnel des populations, et l'idée que seuls les experts, conseillant les gouvernants, étaient à même de nous mener dans la bonne direction $»^{5}$.

Dans cet article, je voudrais examiner ce rapport savoir-pouvoir qui a sous-tendu la gestion de la crise sanitaire par nos Etats. Il repose en effet sur un paradoxe que j'analyserai dans un premier temps. Alors même que la crise du Covid a replongé les scientifiques et les experts dans une incertitude radicale, dans un "moment d'empirisme zéro » pour reprendre les termes de l'historienne Lorraine Daston, une gestion technocratique de la crise s'est mise en place.

6 Dans un second temps, je voudrais explorer une hypothèse qui est au cœur de l'argumentaire du présent Cahier du GRM, suscité entre autres par le contexte de la crise sanitaire. Comme nous l'avons explicité avec Andrea Cavazzini dans l'éditorial, il nous a semblé que ce paradoxe d'une expertise incertaine mobilisée pendant la crise doit être mis en relation avec une dynamique d'«expérimentalisation» continue du monde. L'expérimentation constitue à nos yeux un concept clé pour comprendre la conjoncture de la crise sanitaire ou plutôt celle de la gestion de la crise. L'incertitude a contraint les experts à provoquer des observations, à agir pour produire des connaissances. Je tenterai d'expliciter cette idée que le réel pouvoir des experts réside moins dans l'imposition d'une vérité, que dans le pouvoir d'expérimenter.

7 Dans un troisième temps, j'examinerai les effets politiques de cette dynamique d'expérimentation. Sur le plan méthodologique, je suivrai les indications des travaux de Grégoire Chamayou. Pour lui, il y a une "créativité normative de la pratique expérimentale elle-même, qui vit comme un foyer d'élaboration et d'invention à la fois épistémologique et éthique $»^{6}$. Les expérimentations produisent des effets normatifs. Trois types d'effets me semblent pouvoir être mis en avant: des effets de subjectivation, de temporalisation et de spatialisation.

Dans un dernier temps, je tenterai de baliser les questions ouvertes par notre hypothèse d'une expérimentalisation du monde. Parmi les questions ouvertes par celleci, j'insisterai sur la question politique du pilotage de ces dynamiques d'expérimentation. Si ces dernières ne sont pas sans effets, la question de leur pilotage n'est pas réellement problématisée. La signification de ce qu'on appelle la « démocratie sanitaire » ne se joue-t-elle pas pourtant sur cet enjeu du pilotage de l'expérimentation continue du monde?

\section{Un pouvoir paradoxal des experts}

9 La philosophe Bernadette Bensaude-Vincent soulevait le paradoxe de cette expertise mobilisée pendant la crise :

Les experts, sommés de "dire le vrai au pouvoir », selon la fonction qui leur est traditionnellement attribuée, se trouvent fort dépourvus car ils ne savent presque 
rien sur le Covid-19. S’il est vrai que les méthodes de séquençage ont permis l'identification très rapide du virus, son comportement, les voies de transmission, la période de contagiosité, la durée de l'immunité sont autant d'énigmes qu'il va falloir résoudre ${ }^{7}$.

10 Pour l'historienne des sciences Lorraine Daston, la crise actuelle du Covid-19 nous plonge dans un contexte similaire à celui du XVIIème siècle. Nulle volonté de sa part de comparer le Covid-19 avec la peste. Elle vise plutôt à comparer la situation actuelle de la science face au Covid-19 avec le début de la science moderne :

C'est ce que je veux dire quand j'annonce que nous avons été soudainement catapultés au XVIIe siècle : nous vivons un moment d'empirisme zéro, un moment où presque tout est à inventer, à trouver, comme c'était le cas pour les membres des premières sociétés scientifiques - et tous les autres - vers $1660^{8}$.

11 Ce contexte scientifique n'était pas simplement caractérisé par un manque de connaissance - le progrès scientifique a pour effet d'ouvrir toujours de nouvelles questions - mais par une incertitude méthodologique quant à la manière même de procéder pour acquérir des connaissances.

Derrière l'apparente maîtrise que manifeste l'omniprésence dans les médias de chiffres, statistiques et courbes concernant la maladie, les scientifiques ont été confrontés au début de la crise - et continuent à l'être - à des questions méthodologiques et épistémologique de fond. À titre d'exemple: comment compter les morts liés au Covid-19? Plusieurs approches sont possibles :

Certains pays comptabilisent la mort de toute personne testée positive au Covid-19 comme un décès dû au virus, quels que soient les autres facteurs (comme le diabète, par exemple) qui ont pu jouer un rôle ; d'autres pays prennent en compte la cause dominante ou proche dans leurs classifications; les deux systèmes ont des avantages et des inconvénients 9 .

À lire Lorraine Daston, on se rend compte que le Covid-19, loin de constituer un objet scientifique stabilisé, est encore au cœur de nombreuses controverses à l'interne de chaque discipline scientifique et entres elles.

Malgré l'incertitude scientifique, l'action politique s'est imposée, au risque sinon de voir les conséquences de la crise sanitaire s'aggraver. Les décideurs politiques ont dû alors agir, prendre des mesures, fixer des seuils, des règles, interdire et réguler certaines activités, tout cela sans une connaissance stabilisée et avérée de l'ensemble des paramètres des risques à gérer.

Si on peut s'accorder sur le fait qu'il fallait agir, de plus agir sans connaissances stabilisées et avérées, pourquoi parler néanmoins de "technocratie »? Si je décide d'utiliser ce terme, c'est pour plusieurs raisons. Tout d'abord, c'est parce que, malgré son incertitude d'arrière-fond, l'expertise, en plus d'éclairer les décideurs, a été mobilisée pour son pouvoir de « disciplinement » de la population.

À titre d'exemple, la question du compte des morts a été extraite des controverses scientifiques pour constituer un point de stabilité et un "horizon d'attente " des membres de la société. Comme l'indiquent Catherine Fallon et François Thoreau, alors que le déploiement du virus, instable et mouvant, varie fortement d'un contexte national à l'autre et d'une population à l'autre, l'expertise a été mobilisée pour sa vertu de redonner au politique des balises bien solides pour la planification et l'exécution de mesures aussi fortes que celle du confinement. L'expertise est mobilisée «pour faire comme si nous avions le contrôle et la maîtrise des événements ${ }^{10}$. 

efficacité. Elle a permis de planifier et de faire exécuter des mesures fortes comme le confinement pour éviter le scénario catastrophique d'une saturation de nos hôpitaux. En effet, assez rapidement, s'est posé la question de la gestion des admissions de malades gravement atteints par le Covid au sein des Unités de Soins Intensifs. Outre la question de l'accès au USI, s'est posée également la question de l'allocation des systèmes de ventilation artificielle. Le coronavirus pouvant provoquer une pneumonie aiguë, ce qu'on nomme "syndrome respiratoire aigu sévère ", de nombreux malades risquaient d'avoir besoin de tels systèmes permettant de les maintenir en vie. Un des enjeux a été rapidement d'éviter la saturation des places en USI et une pénurie de respirateurs, entre autres ressources médicales. multiples. Tout d'abord, il occulte le fait que le savoir est pluriel, traversé par des controverses et par des conflits internes aux et entre les disciplines. Jean-Paul Gaudillière, Frédéric Keck et Anne Rasmussen nous rappellent ainsi les différences qu'il existe entre les savoirs produits et les technologies utilisées par la virologie et l'épidémiologie. La première s'est développée comme section particulière de la microbiologie dans les années 70. Les virus sont observés au microscope électronique, leurs mutations suivies par le séquençage génétique. L'épidémiologie, quant à elle, s'est développée dans les années 30, suite à la grippe espagnole: "Elle construit des indicateurs à partir des chiffres déclarés par les autorités sanitaires (taux de contagiosité, taux de létalité...) pour établir des modèles du nombre de cas d'infection dans l'avenir $»^{11}$.

Pour se constituer comme expertise mobilisable par l'action politique, une réduction de ce pluralisme a été effectuée pour se centrer principalement sur une évaluation médico-économique, laissant également largement dans l'ombre les dimensions sociales de la crise sanitaire. Qu'en est-il de l'exploration d'autres scénarii tout aussi catastrophiques? Comme le soulignent certains, « il n'y a pas que le virus qui tue $»^{12}$.

\section{Une expérimentalisation du monde}

16 À côté de ce pouvoir de disciplinement de la population, le pouvoir de l'expertise peut se comprendre également d'une autre façon. Alors que Lorraine Daston a vu dans la crise sanitaire un retour à un moment d'empirisme zéro, où "l'observation, généralement considérée comme le parent pauvre de l'expérience et des statistiques, prend tout son sens ", ne faudrait-il pas plutôt parler de la montée en puissance d'un «moment expérimental », d'un recours généralisé à des observations provoquées?

Comme nous l'évoquions dans l'édito avec Andrea Cavazzini, il nous semble en effet que la gestion de la crise a plutôt pris la forme d'une expérimentation non assumée, d'une dynamique de tâtonnement par essai et erreur qui n'a pas dit son nom. Le mot d'expérimentation est ici choisi à dessein. L'expérimentation en laboratoire est une intervention qui, dans un environnement paramétré et contrôlé, permet de produire de la connaissance. Loin d'une observation distancée et neutre, l'expérimentateur agit pour connaître. De manière analogue, l'agir politique pendant la crise est nécessairement un connaître. Le feedback des mesures permet de réajuster et d'améliorer la gestion du risque. N'est-ce pas une des explications des variations 
continues des seuils et mesures adoptées par les autorités dans un contexte d'incertitude radicale? Bien entendu ces variations peuvent s'interpréter de multiples façons. Pour certains, l'évolution des stratégies des autorités a surtout suivi l'évolution des moyens disponibles ${ }^{13}$. La non-recommandation ou la non-imposition des masques en début de crise aurait découlé ainsi de l'absence de réserve de masques en suffisance. Cette interprétation ne peut-elle pas coexister avec l'hypothèse que la régulation des risques par les autorités a pris la forme d'une expérimentation? Chaque intervention étant indissociablement une action de régulation et un acte de production de connaissance?

Précisons encore notre hypothèse d'une expérimentalisation généralisée en nous arrêtant sur le concept de biopolitique développé par Foucault, mobilisé dans de nombreux articles consacrés à la gestion de la crise. Nul ne pourrait en effet nier le fait que la vie est devenu un enjeu politique et que l'exercice du pouvoir cible la population. Reste à préciser la forme spécifique de la biopolitique de la crise. En écho à la nature paradoxale de l'expertise mobilisée pendant la crise, il faut reconnaître que la biopolitique de la crise repose sur un savoir incertain. Il me semble, plus précisément encore, que la biopolitique de la crise traduit un gouvernement des populations qui est indissociablement une production du savoir qu'elle mobilise. Plutôt qu'une biopolitique qui développe une gestion préventive des risques classique, prenant appui sur une connaissance des maladies passées, la biopolitique de la crise produit de la connaissance. C'est en ce sens qu'on peut parler d'expérimentation. Le disciplinement de la population est en même temps une observation provoquée. Bernadette BensaudeVincent défend, me semble-t-il, une position similaire lorsqu'elle affirme que «le coronavirus crée non seulement une crise mondiale, il transforme le monde en laboratoire ». Plus loin dans son article, elle écrit :

Pour les recherches sur le coronavirus, chaque pays, chaque région offre une cohorte de cas avec des paramètres variables (mesures de confinement, test précoces) qui pourront permettre des comparaisons avec des groupes témoins. Dans ce processus mondial d'apprentissage du contrôle des virus, tous les humains infectés ou pas, traités ou pas, vivants ou morts, deviennent de fait des objets d'expérience, de tests ou d'essais cliniques, des données statistiques. La quête du savoir se confond avec le gouvernement des populations par la biopolitique, et mobilisera sans doute le traçage des individus par leur téléphone portable ${ }^{14}$.

La Commission Nationale Consultative des Droits de l'Homme (France), dans un avis sur le suivi numérique des personnes du 28 avril 2020, rapporte que l'opérateur Orange a partagé des données de localisations anonymisées entre autres avec l'INSERM, afin de permettre à des épidémiologistes de modéliser la propagation de la maladie. «C'est également Orange qui a partagé avec les pouvoirs publics son étude, menée à partir des données de géolocalisation des téléphones de ses abonnés, selon laquelle $17 \%$ des habitants de la métropole du Grand Paris ont quitté leur région entre le 13 et le 20 mars $»^{15}$.

20 Les données étant globalisées et anonymisées, la CNCDH précise que le risque est moins celui d'une mise en danger du respect de la vie privée que celui d'engendrer une défiance de la population à l'égard des autorités et des outils numériques. La mobilisation de ces données de géolocalisation n'atteste-t-elle pas que le monde est devenu un laboratoire?

21 L'usage des technologies numériques pendant la crise peut également se lire, toujours en prenant appui sur Foucault, comme l'articulation particulière des deux pouvoirs sur 
la vie identifiés par Foucault dans le premier tome de l'Histoire de la sexualité. Il y distingue l'anatomo-politique du corps humain de la bio-politique de la population. La première se centre sur le corps comme machine et s'exerce à partir des procédures de pouvoir que constituent les disciplines. La seconde se centre sur le corps-espèce et s'exerce à travers les contrôles régulateurs de la population. Foucault précise encore que ces deux formes du pouvoir sur la vie « ne sont pas antithétiques; elles constituent plutôt deux pôles de développement reliés par tout un faisceau intermédiaire de relations $»^{16}$. Est-ce que les technologies numériques n'articulent pas de manière tout à fait spécifique ces deux formes du pouvoir sur la vie ? Pour Olivier Razac « l'articulation stratégique entre biopolitique et anatomopolitique reste longtemps assez floue, distendue et peu efficace, jusqu'au développement de technologies politiques basées sur les systèmes automatisés, la théorie de l'information, la puissance des ordinateurs et des réseaux de communication $»^{17}$. L'utilisation du téléphone portable, pendant la crise, n'est-il pas tout autant exercice d'une discipline sur les corps, outil de prévention des risques à travers des applications de traçage, qu'outil d'une biopolitique qui produit une connaissance de la contagion du virus au sein de la population? Dans son analyse de la "e-santé », Razac analyse l'articulation de la discipline des corps individuels avec le gouvernement de la vie des populations depuis la perspective d'une cybernétique sociale, les disciplines médiatisées par les outils numériques assurant une fonction de feedback du système. Est-ce que l'on ne peut pas plutôt interpréter cette nouvelle forme de la "technologie à double face $»^{18}$ que constitue le pouvoir à partir du référentiel de l'expérimentalisation généralisée ?

Un dernier indice me semble plaider en faveur de cette interprétation du rapport savoir-pouvoir pendant la crise comme expérimentalisation, à savoir le fait que les autorités publiques revendiquent elles-mêmes le référentiel de l'expérimentation pour encadrer l'innovation technologique. À titre d'exemple, évoquons le livre de Laura Létourneau et Clément Bertholet, intitulé Ubérisons l'Etat! Avant que d'autres ne s'en chargent. Précisons que Laura Létourneau joue un rôle important dans l'évolution du système de santé en France. En effet, elle a été mandatée par l'ancienne ministre de la santé comme "Délégué ministérielle du numérique en santé ». Avec Dominique Pon, elle co-pilote un chantier qui a pour but de déterminer les orientations de la politique du numérique en santé dans le cadre de la stratégie «Ma santé 2022 ». Dans leur livre, Létourneau et Bertholet en appellent à "expérimenter l'action publique ", faisant référence au philosophe pragmatiste John Dewey. L'expérimentation pourrait, selon eux, constituer une réponse à la crise de légitimité de l'action publique. Expérimenter c'est, pour les pouvoirs publics, reconnaître leur ignorance sur des questions inédites, pour pouvoir mener une forme d'évaluation ex ante de certaines politiques avant de les généraliser, et, enfin, confronter les idéologies à l'épreuve des faits: "C'est par l'expérimentation que la puissance publique retrouvera la légitimité d'imposer des choix irréfutables d'intérêt général, ou tout du moins d'écarter des solutions dont l'effet est démontré négatif (...). Expérimenter c'est pour la puissance publique accepter de perdre du terrain aujourd'hui pour mieux réguler ensuite $»^{19}$.

23 Le député Cédric Villani qui a signé un rapport sur l'éthique de l'intelligence artificielle met également en avant la dynamique de l'expérimentation comme modalité de l'évaluation de l'IA :

Afin de bénéficier des avancées de l'IA en médecine, il est important de faciliter les expérimentations de technologie IA en santé en temps réel et au plus près des usagers, en créant les conditions réglementaires et organisationnelles nécessaires 
(" un bac à sable»). Ce bac à sable aurait pour objectif de faciliter les démarches d'expérimentation, de conception itérative et à terme de déploiement des technologies d'IA en santé avec les usagers (hôpitaux, professionnels de santé, patients, etc.). En effet, l'expérimentation « en condition réelle » est indispensable pour tester l'efficacité du système et l'améliorer en se fondant sur les retours

d'expérience et les données produites en situation par les usagers ${ }^{20}$.

24 s'est rapidement étendue à l'échelle de la société tout entière et ce, bien souvent de manière non réellement contrôlée. A titre d'exemple, de nombreux avis et rapports de comités éthiques ont mis en avant le fait que des applications de suivi numérique des personnes ont été implémentées sans réelle connaissance tant de leur efficacité sanitaire que de leurs multiples effets sociaux. En France, le Comité National Pilote d'Ethique du Numérique soulignait ainsi l'importance d'expérimenter au préalable les applications de traçage, tout en soulignant que les expérimentations préalables au lancement de l'application qui ont été menées dans un environnement contrôlé se heurtent bien souvent à des limites de choix et de taille de l'échantillon, de même qu'à des limites de temps pour les conduire. Pour lui, une expérimentation précipitée et une validation insuffisante pourraient avoir pour effet de nuire à l'efficacité de l'application et d'induire par exemple un débordement du système de tests médicaux par des faux positifs (notifiés mais testés négativement par la suite). L'inefficacité de l'application pourrait également avoir pour effet d'affecter la confiance dans la gestion de la crise. En conclusion, le CNPEN recommande de poursuivre les expérimentations en parallèle $\mathrm{du}$ déploiement de l'application: "Si une application est déployée, il serait donc souhaitable, pour pouvoir la corriger et l'améliorer, de poursuivre les expérimentations durant le déploiement afin de prendre en compte leurs résultats en même temps que les retours d'expérience de ce déploiement $»^{21}$.

\section{La créativité normative de l'expérimentation}

Poursuivons maintenant l'analyse des implications de l'hypothèse d'une montée en puissance d'une expérimentalisation du monde pendant la crise. Pour ce faire, je m'inspire de la méthodologie de recherche suivie par Grégoire Chamayou dans son livre Les corps vils, Expérimenter sur les êtres humains aux XVIIIème et XIXème siècle. Dans ce livre, il mène une recherche historique et épistémologique portant sur les dispositifs expérimentaux. Il conduit cette recherche en s'intéressant à une histoire bien souvent occultée par les historiens des sciences, à savoir celle des sujets de l'expérience, ou plutôt faudrait-il dire l'histoire des formes de subjectivation des sujets de l'expérience. En effet, pour lui, le développement des dispositifs expérimentaux est indissociable de l'acquisition de corps. Cette chasse aux corps sur lesquels expérimenter est rendue possible par des technologies d'avilissement des corps. Des subjectivités sont avilies pour en permettre l'expérimentation.

Refusant la position d'une éthique de la recherche abstraite qui poserait la question des sujets de l'expérience de manière générique et universelle - l'expérimentation sur l'Homme -, il tente plutôt de montrer, au fil de son enquête, combien l'expérimentation a été rendue possible par un travail de qualification ou plutôt de disqualification de certaines catégories de corps comme vils, légitimant ainsi leur soumission à l'expérimentation. L'histoire des dispositifs d'expérimentation est indissociable de l'histoire des corps des suppliciés, des condamnés, des colonisés, des indigents, etc. 
Expérimenter, c'est donc exercer un effet de subjectivation, soit que l'expérimentateur contribue directement à qualifier un corps de corps vil, soit qu'il relaye, consciemment ou inconsciemment, certaines disqualifications sociales. Sur le plan épistémologique, la posture de Chamayou est inverse à celle d'un jugement déterminant. Il ne s'agit en aucune façon de chercher à « encadrer " l'expérimentation, à subsumer sous une règle ou un principe une expérimentation particulière. Au contraire, il s'agit plutôt de considérer que les pratiques expérimentales produisent des effets normatifs. Le renouvellement de la pratique expérimentale, la production de nouvelles techniques et méthodologies, créent des problèmes éthiques et de nouveaux sujets éthiques :

L'image d'un cadre normatif est réductrice: il n'y a pas entre la pratique expérimentale et le discours éthique un rapport comparable à celui d'un tableau entouré par un cadre, chaque élément pouvant être modifié séparément, extérieurement l'un à l'autre; il y a au contraire une créativité normative de la pratique expérimentale elle-même, qui vit comme un foyer d'élaboration et d'invention à la fois épistémologique et éthique ${ }^{22}$.

Cette considération d'une créativité normative de l'expérimentation va nous conduire à poursuivre l'analyse des implications de l'hypothèse d'une montée en puissance d'une expérimentalisation du monde pendant la crise en abordant les effets de cette dynamique d'interprétation. Si le livre de Chamayou met surtout l'accent sur l'effet de subjectivation produit par les expérimentations, il me semble que cette analyse peut être complétée par une investigation des effets de temporalisation et de spatialisation induits par l'expérimentalisation du monde pendant la crise.

Précisons encore qu'identifier et analyser réellement ces effets requerrait un travail d'enquête de terrain. Rappelons que Chamayou, dans son livre, mène une enquête historique portant sur les expérimentations sur les êtres humains aux XVIIIème et XIXème siècle. Les effets des dispositifs expérimentaux ont donc laissé des traces qu'une enquête rigoureuse peut suivre. Dans l'actualité de la crise, les effets d'une expérimentalisation du monde pendant la crise sont plus difficilement visibles et demanderaient d'autres disciplines scientifiques que l'histoire pour être étudiés. Je me limiterais donc ici à soulever quelques questions pour baliser l'enquête sur ces effets.

\section{Effets de subjectivation induits par l'expérimentalisation du monde}

Comme l'indiquent également David, Godrie et Benhmarhnia dans leur ouvrage collectif intitulé Les sociétés de l'expérimentation, Enjeux épistémologiques, éthiques et politiques, l'expérimentation est une «modalité de production des savoirs transformant les sujets et les objets qu'elle met en relation $»^{23}$. Une première manière de comprendre cette idée d'une transformation des sujets est de la relier à la question classique de l'échantillon retenu pour une expérimentation. Les expérimentations menées sur un échantillon de population permettent-elles de généraliser les résultats à la population cible (target population)?

En sciences médicales, le gold standard de l'expérimentation prend la forme de l'essai contrôlé randomisé (ECR). La méthodologie de ce type d'essai cherche à isoler l'effet causal de l'expérimentation (un médicament, etc.) :

Dit autrement, on cherche à mettre en évidence le fait que les changements observés à la suite d'une implantation de l'expérimentation sont bel et bien attribuables à l'expérimentation et non pas à des sources exogènes, ce qui renvoie à 
une démarche d'inférence causale (...). Les ECR ont été mis en place pour répondre à cette démarche d'inférence causale et sont particulièrement intéressants de ce point de vue, ce qui peut expliquer leur popularité, et ainsi leur déploiement dans de nombreux champs d'intervention ${ }^{24}$.

31 Après en avoir déterminé la finalité, reste à préciser comment fonctionne ce gold standard. Un des modèles les plus courants consiste à comparer deux groupes supposés identiques. Le premier groupe - le groupe factuel - reçoit une intervention. Un second - le groupe contrefactuel - se distingue du groupe factuel par le seul fait de n'avoir pas reçu l'intervention. L'effet causal est donc mesurable par le contraste entre ces deux groupes. La randomisation est utilisée pour obtenir un groupe contrefactuel valide. Une «répartition intentionnelle et aléatoire vis-à-vis de la probabilité de recevoir le traitement permet de s'assurer qu'il n'y a pas de différences systématiques entre le groupe traitement et le groupe témoin avant de recevoir l'intervention, générant ainsi les conditions d'obtenir un substitut valide du groupe contrefactuel ${ }^{25}$.

Bien d'autres éléments sont nécessaires pour inférer un effet causal et pour prendre en compte les «facteurs de confusion", c'est-à-dire les caractéristiques pouvant influencer la probabilité de recevoir le traitement ou une intervention. Ce qui m'intéresse ici, c'est d'insister sur une limite épistémologique de ce modèle. Pour Nancy Cartwright, ce gold standard est caractérisé par une étroitesse d'esprit (narrowness of scope): «RCT's have high internal validity but the formal methodology puts severe constraints on the assumptions a target population must meet to justify exporting a conclusion from the test population to the target $»^{26}$. La validité de son inférence causale est interne à la population de l'expérimentation et peut plus difficilement être étendue à la population cible, c'est-à-dire la population pour laquelle les conclusions de l'expérimentation sont censées être utilisées. Comme l'écrit Tarik Benhmarhnia :

Par exemple, si une expérimentation est menée pour tester l'efficacité d'un revenu universel inconditionnel dans une région québecquoise comme la Montérégie, la population cible pourrait être la population québecquoise ou canadienne dans son ensemble. Il est néanmoins facile d'imaginer qu'une expérimentation dans un contexte donné a peu de chances de représenter un échantillon aléatoire de la population cible. En effet, la population de l'expérimentation et la population cible peuvent différer vis-à-vis de caractéristiques observables (genre, sexe, âge, niveau d'éducation, etc.), mais aussi non observables, ce qui entrave la validité des extrapolations ${ }^{27}$.

33 Ces réflexions épistémologiques ne sont-elles pas indissociablement normatives? Le choix de la population de l'expérimentation ne va-t-elle pas contribuer à façonner la population cible, si la mesure ou la politique expérimentée est généralisée? De plus, quel rôle l'expérimentation attribue-t-elle aux participants de l'expérimentation ? Ces derniers constituent-ils des parties prenantes actives de l'expérimentation ou de simples objets sur lesquels expérimenter? L'expérimentation vise-t-elle à associer patients et professionnels à l'élaboration de l'expérimentation ? Un savoir d'expérience est-il reconnu aux patients ou leur implication dans les équipes de recherche a-t-elle plutôt pour finalité de faciliter le recrutement des participants aux essais ${ }^{28}$ ?

34 Pendant la crise sanitaire, la population n'a-t-elle pas été réduite à l'objet d'une expérimentation, alors que des forces de société civile auraient pu contribuer à l'intelligence de l'expérimentation? Nicolas Henckes ${ }^{29}$, dans un numéro de la revue Esprit, nous rappelait le rôle d'enquêteur qu'ont joué les mouvements sociaux dans les champs du handicap, de la santé mentale ou du Sida. Loin de se réduire à des groupes d'intérêts représentants les seuls intérêts des malades, ces mouvements ont contribué à 
l'amélioration de la science et des pratiques médicales. Ces mouvements n'auraient-ils pas permis de produire des perceptions alternatives des conséquences de la pandémie et des effets des mesures de gestion de la crise?

\section{Effets de temporalisation induits par l'expérimentalisation du monde}

Un deuxième type d'effet normatif touche à la temporalité des expérimentations. Quand doit commencer et se terminer une expérimentation? Quelle est sa durée adéquate? Ces questions méthodologiques ne sont pas sans incidence éthique. Une expérimentation trop courte ne permettra qu'un retour limité de la part des usagers. Comme je l'évoquais plus haut, dans son avis portant sur les applications de tracing utilisé dans le cadre de la crise sanitaire, le Comité national pilote d'éthique du numérique met en avant le risque de valider les applications de traçage par une expérimentation précipitée. Dans un autre bulletin de veille consacré intitulé "Réflexions et points d'alerte sur les enjeux d'éthique du numérique en situation de crise sanitaire aigue ", le CNPEN affirmait qu'«il faut cependant s'interroger dès à présent sur la mutation sociétale que la généralisation de ces innovations engendrerait à terme $»^{30}$.

Un décalage se donne à voir entre la temporalité courte des expérimentations contrôlées et le pouvoir de transformation sociale des technologies sur un temps plus long. Afin de saisir ce point, il faut réaliser qu'utiliser des technologies comme celles utilisées pour le suivi numérique et, de manière générale, utiliser une technologie quelle qu'elle soit, c'est introduire une inactualité dans le temps social. Pour le philosophe des techniques Xavier Guchet, les médiations techniques inscrivent au sein des groupes humain l'inactualité d'un passé mais aussi celle d'un futur. Guchet reprend les analyses de Michel Serres pour qui nous ne sommes pas contemporains de nos techniques :

Un véhicule automobile est un feuillage de temporalités hétérogènes, depuis celle de la roue dont "l'invention » se perd dans un passé multimillénaire, jusqu'à celle des systèmes électroniques les plus sophistiqués ou des matériaux composites ${ }^{31}$.

De plus, « la médiation technique inscrit au sein des groupes humains l'inactualité d'un passé mais aussi celle d'un futur " "2 $^{2}$ Les technologies rendent le futur « déjà là », nous dit Guchet : «Les techniques introduisent à (...) une ontologie prospective : l'étoffe du monde a un élément de prospective, non pas parce qu'il contient des promesses et que des scénarios sont écrits, mais parce que le futur est déjà là, dans les configurations présentes et en devenir de la technique $»^{33}$.

Une autre manière encore de comprendre cette inactualité des technologies est de réaliser que leurs effets ne se donnent pas toujours à voir dans la temporalité courte de leur usage actuel. Ces effets peuvent se manifester sur une temporalité plus longue. Guchet nous rappelle ainsi cette leçon de Henri Bergson :

Un siècle a passé depuis l'invention de la machine à vapeur et nous commençons seulement à ressentir la secousse profonde qu'elle nous a donnée. La révolution qu'elle a opérée dans l'industrie n'en a pas moins bouleversé les relations entre les hommes. Des idées nouvelles se lèvent, des sentiments nouveaux sont en voie d'éclore ${ }^{34}$. 
38 À ce que Guchet qualifie de «distorsion temporelle principielle du technique et du social ", l'éthique contemporaine des technologies a donné la forme d'un dilemme, plus connu sous le nom de dilemme de Collingridge, du nom de l'auteur de The Social Control of Techology (1980). Ce dilemme consiste en ce que l'anticipation des impacts sociaux d'une technologie est difficile tant que celle-ci n'a pas été développée, car ses effets sociaux ne sont pas encore visibles. Par contre, dès que celle-ci est développée et largement utilisée, il devient très difficile de faire marche arrière et de la modifier. Entre information et pouvoir, il faut choisir.

39 L'utilisation de nouvelles technologies expérimentales à l'échelle de la société pendant la crise introduit donc un pouvoir de transformation sociale qui ne se manifeste pas dans la temporalité courte de l'urgence. C'est un point que j'ai également mis en avant dans un article consacré à la généralisation de la télémédecine en contexte d'urgence sanitaire ${ }^{35}$. Qu'est-ce que la télémédecine ? L'OMS la définit de la manière suivant en 1998 : « La télémédecine clinique est une activité professionnelle qui met en œuvre des moyens de télécommunications numériques permettant à des médecins et d'autres membres du corps médical de réaliser à distance des actes médicaux pour des malades $»^{36}$. Face à la crise du coronavirus, cette médecine à distance a été fortement encouragée, et de manière unanime, par différentes autorités. Elle devait permettre d'évaluer à distance les patients potentiellement affectés sans les faire venir dans un cabinet, de même qu'elle devait constituer un dispositif essentiel pour garantir la continuité des soins aux autres patients, particulièrement aux patients malades chroniques.

40 Un récent rapport du KCE sur la téléconsultation rappelle que «jusqu'à l'entrée en vigueur des mesures temporaires introduites en mars 2020 dans le cadre de la pandémie du Covid-19, il n'existait pas de législation spécifique pour définir et organiser le recours à la vidéo-consultation en Belgique ${ }^{37}$. Mi-mars 2020, le gouvernement a alors publié un Arrêté Royal ${ }^{38}$ pour permettre à tous les médecins de dispenser des conseils téléphoniques à leurs patients, dans le but d'effectuer un «tri » et du renvoi des cas probables de Covid-19, et afin de garantir la continuité des soins des autres patients. Des nouveaux codes de nomenclature ont été introduits pour prévoir le remboursement de ces prestations (vidéo-consultations inclues).

41 En France, la situation était tout autre. Alors qu'en Belgique, il n'existait pas avant la crise de législation spécifique à la télémédecine, un cadre règlementaire de la télémédecine existe déjà en France depuis plus d'une dizaine d'année. Face à la crise, les pouvoirs publics français ont pris des mesures dérogatoires de ce cadre d'exercice de la télémédecine et des conditions de son remboursement pour faciliter la continuité de soins. Le décret $\mathrm{n}^{\circ}$ 2020-227 du 9 mars 2020 a ainsi voulu faciliter l'exercice de la télémédecine en énonçant des « conditions dérogatoires permettant la prise en charge par l'assurance maladie de la téléconsultation pour les patients atteints ou potentiellement infectés par le Covid-19 ${ }^{39}$.

Le point commun est donc que, tant en Belgique qu'en France, un cadre provisoire et souple d'exercice de la télémédecine a été mis en place pour assurer le remboursement des actes de téléconsultation et permettre leur généralisation pendant ce temps de crise. Un point commun est la possibilité qu'ouvrent ce décret et cet Arrêté d'utiliser des applications grand public. En France, le décret n ${ }^{\circ} 2020-227$ du 9 mars 2020 a autorisé les professionnels à titre dérogatoire à télé-consulter via des outils numériques de communication "grand public», s'ils ne disposaient pas des 
équipements nécessaires à l'utilisation de dispositifs référencés et sécurisés. En Belgique, même si une Task Force "Data \& Technology against Corona » a recommandé certains plateformes, une certification officielle de celles-ci n'existe pas encore.

Face à cette utilisation massive de technologies numériques non certifiées, le sociologue du numérique Antonio Casilli nous mettait en garde dès mars 2020 :

Le numérique qu'on nous enjoint de pratiquer en ce moment pourrait ainsi être qualifié de quick and dirty, « rapide et sale »: des solutions adoptées en catastrophe et qui peuvent poser au mieux des problèmes sérieux en termes de surveillance, gouvernance et propriété des données produites; et au pire qui nous exposent à tout un tas de risques qu'on n'a pas encore envisagés ${ }^{40}$.

Un autre article met en avant le fait que la diffusion massive de technologies de basse qualité dans le domaine de la santé mentale aura sans doute rendu des services à court terme, mais impactera durablement le système de santé à long terme. :

In the U.S., the Food and Drug Administration relaxed regulation of mental health apps for depression, anxiety and insomnia in order to facilitate expanded use of digital health tools during the pandemic. Lowering standards may lead to efficiency in the short-term but the widespread adoption of low-quality technology during the pandemic could lead to a long-term substandard tier of service ${ }^{41}$.

L'usage expérimental de technologies a donc des effets qui se lisent sur un plan proprement temporel. Ces effets, à nouveau, ne sont pas neutres, mais éthiques et politiques.

\section{Effets de spatialisation induits par l'expérimentalisation du monde}

La montée en puissance d'une dynamique d'expérimentalisation pendant la crise a également des effets sur le plan spatial. Produire du savoir de manière expérimentale transforme les espaces. Ces effets gagneraient à être étudiés. On pourrait tout d'abord se dire qu'une démarche expérimentale est la garantie de porter attention à la particularité du contexte. Si la démarche d'expérimentation invite à prendre en compte les réalités contextuelles locales, l'objectif reste bien souvent la "scalabilité ", un terme que l'anthropologue Anna Lowenhaupt Tsing définit comme «la capacité des projets à s'étendre sans que le cadre de leur hypothèse ne change $»^{42}$ : on expérimente localement certes, mais semble-t-il pour dégager ce qui est généralisable et opérationnel de manière indifférenciée. N'est-ce pas le cas dans les démarches d'expérimentation à l'œuvre dans l'évaluation de technologies émergentes : on s'ouvre à l'imprévu et à la découverte dans l'expérimentation locale, mais dans le but de dégager ce qui est généralisable et maîtrisable à d'autres échelles ?

Pour problématiser ces effets spatiaux, l'exemple du suivi numérique peut à nouveau être mobilisé ici. Le risque soulevé par le Comité National Pilote d'Ethique du Numérique de généraliser une application de traçage après une expérimentation précipitée et une validation insuffisante ne doit pas être compris uniquement en référence aux limites de l'échantillon, de même qu'à des limites de temps pour conduire les expérimentations. Ne faut-il pas également comprendre ce risque en relation avec l'espace dans lequel ont été menées les expérimentations ? Cet espace estil généralisable? Tous les espaces s'équivalent-ils?

L'usage intensif et généralisé de technologies numériques aura fait ressurgir, tel un refoulé, la question de la spatialitée ${ }^{43}$ que l'on pensait avoir maîtrisé grâce à ces 
technologies. Il n'est pas rare d'entendre que les technologies de l'information et de la communication auraient pour effet de réduire les distances et de maitriser la variable spatiale de l'existence humaine. La témélédecine n'a-t-elle pas permis d'assurer la continuité des soins malgré cette expérience de confinement généralisé de la population? Or, "plusieurs travaux (...) s'attachent à montrer que l'usage de la télémédecine ne conduit pas à une abolition des frontières et des espaces, contrairement à une vision du sens commun souvent portée implicitement par les politiques publiques ${ }^{44}$. Bien au contraire, pour s'effectuer les actes de télémédecine demandent un certain réaménagement des espaces. Qu'en est-il des personnes vivant à plusieurs dans des appartements trop exigus? Comment reproduire les conditions d'intimité comparable à celles d'un cabinet médical? Tous les lieux de vie ne s'équivalent pas. La généralisation sociale de résultats d'une expérimentation locale prend-elle en compte cette hétérogénéité des espaces?

Les effets des expérimentations gagneraient également à être questionnés sur le plan de la «multiscalarité » qui les sous-tend. Par ce terme, j'entends faire référence aux travaux du géographe Michel Lussault qui nous invite à penser la spatialité comme une connexion de différentes " tailles spatiales ${ }^{45}$. Une expérimentation ne transforme pas seulement les sujets, elle n'ouvre pas seulement à des temporalités hétérogènes, elle connecte également différentes échelles spatiales. Une telle articulation n'est pas sans effets de pouvoir. L'emboîtement de différentes échelles spatiales, peut impliquer une hiérarchisation et une asymétrie en termes de contrôle et de pouvoir décisionnel.

L'attention portée aux jeux d'échelle permet de prendre conscience qu'une dynamique d'enquête collaborative au niveau local peut coexister avec une politique centralisée et autoritaire, dans la mesure où c'est cette dernière qui définit les lieux et les échelles où certaines pratiques peuvent s'appliquer et qui décide en dernière instance de la possibilité de les généraliser par-delà leur émergence locale. Dans son livre sur $L a$ grande santé, Olivier Razac rappelle cet effet d'articulation des sphères privée et publique des technologies sociales propres aux sociétés néolibérales ${ }^{46}$. Il cite aussi à ce sujet Robert Castel: "Cette stratégie qui tente de conjuguer la planification centralisatrice et l'initiative privée, l'autoritarisme des technocrates et la convivialité des associations spontanées de citoyens, l'objectivité qu'on prête aux professionnels et les bons sentiments qui sont censés être l'apanage des bénévoles $»^{47}$.

Penser le pouvoir à partir des jeux d'échelles d'une production expérimentale du savoir permet enfin de dépasser une vision simpliste de la technocratique en termes de centralisation et verticalisation des décisions. Le pouvoir des experts est souvent associé à un exercice du pouvoir centralisé et à un rapport vertical par rapport à la population. Or il est indéniable que «lors du confinement, c'est la créativité et la capacité d'auto-organisation des personnels exposés (soignants, caissières, éboueurs, etc.) qui, davantage que les décisions régaliennes, a permis de modifier le scénario écrit à l'avance d'une catastrophe sans retour $~^{48}$. L'hypothèse d'une interprétation du rapport savoir pouvoir au cœur de la gestion de la crise en termes d'expérimentaliation, ne permet-elle pas de rendre visible la forme technocratique particulière qu'a prise l'exercice du pouvoir? Celle-ci ne se donne-t-elle pas à voir comme une forme de pilotage centralisé de dynamiques d'innovations locales décentralisées? 


\section{Conclusion}

Cet article repose sur une hypothèse heuristique, à savoir que le rapport savoir-pouvoir qui a sous-tendu la gestion de la crise peut se comprendre comme une dynamique d'expérimentalisation du monde. Plusieurs questions restent entières face à cette hypothèse. Tout d'abord, poser une telle hypothèse ne conduit-il pas à produire une image trop homogénéisée des expertises mobilisées? Plusieurs formes de rapport savoir-pouvoir ont coexisté pendant la crise. La poursuite de la recherche autour de cette hypothèse devrait donc se centrer sur l'étude de cas d'études concrets et spécifiques, comme je l'ai ébauché avec le cas des applications de suivi numérique et la télémédecine, afin d'étudier avec précision le type d'expertise produite et mobilisée.

51 Une autre question plus massive peut être adressée à l'hypothèse centrale de cet article : s'agit-il vraiment d'expérimentation ? Les phénomènes que recouvre le concept d'expérimentation ne doivent-ils pas plutôt être compris comme l'indice d'une «stratégie du choc » théorisée par Naomi Klein? Dans un article du 13 mai 2020, paru dans The Guardian ${ }^{49}$, elle rapporte les propos suivants de Eric Schmidt, CEO de Google, qui avait pris la direction d'un groupe d'experts chargé d'inventer l'avenir post-Covid dans l'Etat de New York.

The first priorities of what we're trying to do are focused on telehealth, remote learning, and broadband (...) We need to look for solutions that can be presented now, and accelerated, and use technology to make things better (...). The need for fast, large-scale experimentation will also accelerate the biotech revolution (...) Finally, the country is long overdue for a real digital infrastructure (...) If we are to build a future economy and education system based on tele-everything, we need a fully connected population and ultrafast infrastructure.

Si le vocabulaire de l'expérimentation est mis en avant par Schmidt lui-même, ne s'agit-il pas plutôt d'une nouvelle illustration de la stratégie du capital qui profite des grandes catastrophes pour faire passer des réformes ultralibérales? Naomi Klein écrit ainsi qu'une "pandemic shock doctrine" est en train d'émerger, une doctrine qu'elle qualifie de «Screen New Deal». L'expérimentation ne serait-elle qu'une métaphore pour parler de cette stratégie du choc? En réponse à ces questions, il faudrait mettre en avant que ces stratégies capitalistes ont bien partie liées avec une posture expérimentale, au sens où il s'agit de tester, à l'échelle de la société, de nouveaux dispositifs et technologies.

Si la crise constitue bien évidement une opportunité pour certains acteurs économiques, il me semble que le « choc » est tout autant créé par la crise sanitaire que par les mesures entreprises pour y répondre. Les stratégies capitalistes et l'expérimentalisation du monde gagneraient à être analysées ensembles. Sur ce point encore, les recherches de Chamayou sur l'expérimentation sont très inspirantes. Il nous rappelle que Marx lui-même dans Le Capital assimile les changements dans les procédés de production à des expérimentations, les ouvriers constituant des corps vils qui subissent des expériences. Le grand expérimentateur moderne, c'est avant tout le capital, écrit Chamayou ${ }^{50}$.

Enfin, une dernière question me semble importante à investiguer dans la suite de cet article. Au-delà d'une enquête sur les effets de la production expérimentale de l'expertise, est-il possible d'ouvrir la question de son pilotage? La forme technocratique de cette production de savoir est-elle inéluctable ? Ce que l'on appelle la 
« démocratie sanitaire » en France ne gagnerait-elle pas à s'étendre à ces enjeux ? Pour Barbara Stiegler, le concept institutionnel français de "démocratie sanitaire " est aujourd'hui tiraillé entre deux conceptions incompatibles de la démocratie :

La première, conforme au modèle lippmannien du gouvernement des experts, renforce la dichotomie entre une expérimentation. Menée d'en haut par les experts et la cible de l'expérimentation: une population réduite au « consentement » et à «l'acceptabilité sociétale des innovations». L'autre, beaucoup plus proche de la démocratie participative théorisée par Dewey, entend réinterpréter la « démocratie sanitaire" comme l'occasion d'une participation active des public à l'expérimentation, à la production des connaissances et au choix des objectifs collectifs en matière de santé publique ${ }^{51}$.

La gestion technocratique de la recherche expérimentale de solutions était-elle inéluctable? Autant de questions qui restent entières et sur lesquelles ces recherches sur l'expérimentation devraient se poursuivre.

\section{NOTES}

1. Anne-Emmanuelle Bourgaux, «Le Covid-19, crash-test pour la démocratie belge», in Démocratie, juin 2020.

2. Marc Maesschalck, "Pandemia y filosofia política », traduit par Aníbal Pineda Canabal, in Escritos 28, 60, 2020, pp. 125-132.

3. Oihana Gabriel, «Coronavirus : Les associations de patients ne veulent plus être laissées de côté », https://www.20minutes.fr/sante/2856715-20200908-coronavirus-associations-patientsveulent-plus-etre-laissees-cote

4. Bernadette Bensaude-Vincent, «Penser l'après: Sciences, pouvoir et opinions dans l'après Covid-19 ", in The conversation, 2 mai, 2020, https://theconversation.com/penserlapres-sciences-pouvoir-et-opinions-dans-lapres-covid-19-137272

5. Barbara Stiegler, «Faire face aux experts. Néolibéralisme et pandémie », in Terrestres, 26 juin 2020, https://www.terrestres.org/2020/06/26/faire-face-aux-experts-neoliberalisme-etpandemie/.

6. Grégoire Chamayou, Les corps vils. Expérimenter sur les êtres humains aux XVIIIe et XIXe siècles, Paris, La Découverte, 2009, p. 226.

7. B. Bensaude-Vincent, op. cit.

8. Lorraine Daston, "Covid-19 ou le degré zéro de l'empirisme », in AOC, 29 avril 2020, https:// aoc.media/opinion/2020/04/28/covid-19-ou-le-degre-zero-de-lempirisme/

9. Ibid.

10. Catherine Fallon et François Thoreau, "Savoirs, risques et expertises ", in Politique, juillet 2020, n 112, p. 21.

11. Jean-Paul Gaudillière, Frédéric Keck, Anne Rasmussen, « Des virus, des humains, des savoirs, des épidémies : la construction sociale de quoi ? ", in Newsletter de l'EHESS, https://www.ehess.fr/ $\mathrm{fr} /$ carnet/coronavirus/virus-humains-savoirs-épidémies-construction-sociale-quoi

12. Adrien Renaud, "Attention à la bombe à retardement post-épidémie ", in Les GénéralistesCSMF. Le syndicat de tous les spécialistes en médecine générale, 27 avril 2020 : https://lesgeneralistescsmf.fr/2020/04/27/attention-a-la-bombe-a-retardement-post-epidemie-les-medecins-face-auxdommages-collateraux-du-covid-19/ 
13. Catherine Fallon, Aline Thiry, Sébastien Brunet, «Planification d'urgence et gestion de crise sanitaire. La Belgique face à la pandémie de Covid-19", in Courrier hebdomadaire du CRISP, 2020/8-9 (n 2453-2454), pp. 5-68 p. 46.

14. Bernadette Bensaude-Vincent, op. cit.

15. https://

www.cncdh.fr/sites/default/files/avis_2020_-_3_-

_200424_avis_suivi_numerique_des_personnes.pdf

16. Michel Foucault, Histoire de la sexualité I, La volonté de savoir, Paris, Gallimard, 1976, pp. 182-183.

17. Olivier Razac, La grande santé, Paris, Flammarion, 2006, p. 198.

18. Michel Foucault, Histoire de la sexualité I. La volonté de savoir, op. cit., 183.

19. Clément Bertholet et Laura Létourneau, Ubérisons l'Etat, Avant que d'autres ne s'en chargent, Paris, Armand Colin, 2017, pp. 186-187.

20. Cédric Villani, Donner un sens à l'intelligence artificielle, 2018, p.199, https:// www.aiforhumanity.fr/pdfs/9782111457089_Rapport_Villani_accessible.pdf

21. https:// www.ccne-ethique.fr/fr/actualites/cnpen-enjeux-dethique-concernant-des-outilsnumeriques-pour-le-deconfinement.

22. Grégoire Chamayou, Les corps vils. Expérimenter sur les êtres humains aux XVIIIe et XIXe siècles, Paris, La Découverte, 2009, p. 226.

23. Tarik Benhmarhnia, Pierre-Marie David et Baptiste Godrie (éds.), Les sociétés de l'expérimentation, Enjeux épistémologiques, éthiques et politiques, Québec, Presses de l'Université du Québec, 2019, p. 3.

24. Tarik Benhmarhnia, «Pourquoi aime-t-on tant les essais contrôlés randomisés? Les attraits et les limites de l'expérimentation », in T. Benhmarhnia, P.-M. David et B. Godrie (éds.), Les sociétés de l'expérimentation, Enjeux épistémologiques, éthiques et politiques, op. cit., pp. 61-77, p 62.

25. Ibid., p. 63.

26. Nancy Cartwright, « Are RCTs the gold standard », in BioSocieties, vol 2, n 1, pp. 11-20, p. 4.

27. T. Benhmarhnia, « Pourquoi aime-t-on tant les essais contrôlés randomisés ?», op. cit., p. 65.

28. A. Hughes-Morley et al., " The impact of advertising patient and public involvement on trial recruitment: Embedded cluster randomized recruitment trial », in Trials, vol. 17, pp. 586-599.

29. Nicolas Henckes et al., «La démocratie à l'épreuve de l'épidémie », in Esprit, octobre 2020, p. 102.

30. https://www.ccne-ethique.fr/sites/default/files/publications/bulletin-1-ethique-dunumerique-covid19-2020-04-07.pdf

31. Xavier Guchet, Philosophie des nanotechnologies, Paris, Hermann, 2014, p. 21.

32. Ibidem.

33. Ibid., p. 22.

34. Henri Bergson, L'Evolution créatrice, Paris, PUF, 1941, p. 139.

35. Alain Loute, "Télémédecine et crise sanitaire. Réflexions éthiques et politiques ", in Revue Démocratie, $\mathrm{n}^{\circ} 10$, octobre 2020, pp.4-8: http://www.revue-democratie.be/index.php? option=com_content\&view=article\&id=1452:telemedecine-et-crise-sanitaire-reflexions-ethiqueset-politiques\&catid=30\&Itemid=130.

36. Pierre Simon, «Définitions et apports de la télémédecine pour la santé publique », in Actualité et dossier en santé publique, décembre 2017, n 101, p. 10.

37. Federaal Kenniscentrum voor de Gezondheidszorg (KCE) https://kce.fgov.be/sites/default/ files/atoms/files/

KCE_328B_videoconsultations_pour_maladies_chroniques_somatiques_Synthese.pdf .

38. https://www.etaamb.be/nl/koninklijk-besluit-van-18-maart-2020_n2020040672.html

39. Lina Williatte-Pellitteri, « Regard juridique sur l'avènement de la télé-santé lors de la crise sanitaire », in RDS, n 96, 2020. 
40. Antonio Casilli, "Interview avec Raphaël Bourgois ", in AOC, 28 mars 2020, http:// www.casilli.fr/2020/03/28/pour-sortir-de-cette-crise-pandemique-il-faut-abattre-lasurveillance-de-masse-grand-entretien-aoc-28-mars-2020/

41. N. Martinez-Martin, I. Dasgupta, A. Carter, J. A. Chandler, P. Kellmeyer, K. Kreitmair, A. Weiss, L.Y. Cabrera, «Ethics of Digital Mental Health During COVID-19: Crisis and Opportunities » in JMIR Ment Health ,2020, 7(12), e23776.

42. Anna Lowenhaupt Tsing, Le champignon de la fin du monde, Sur la possibilité de vivre dans les ruines du capitalisme, Paris, La Découverte, 2017, p. 78.

43. Sur cet enjeu de la spatialité en relation au numérique en santé, le lecteur pourra se rapporter aux articles suivants: Alain Loute, «De l'éthique de l'accueil à l'éthique de la spatialisation des soins ", in E. Clarizio, R. Porta et M. Spano, Milieu, mi-lieu, milieux, Milan, Editions Mimesis, 2020, pp. 243-264; Alain Loute, «La spatialité au cœur des éthiques de l'hospitalité et des éthiques du soin ", in S. Bourgault, S. Cloutier et S. Gaudet (dir.), Ethiques de l'hospitalité, du don et du care: Actualités, regards croisés, Ottawa, Les Presses de l'Université d'Ottawa, 2020, pp.19-39; Alain Loute, «Spatialisation et santé numérique : quels défis pour l'éthique?", in C. Hervé, M. Stanton-Jean et M. Deschênes (éds.), Les personnes âgées et le numérique, Paris, Dalloz, 2019, pp. 81-94.

44. A. Mathieu-Fritz et G. Gaglio, "À la recherche des configurations sociotechniques de la télémédecine. Revue de littérature des travaux de sciences sociales ", in Réseaux 2018/1, n²07, p. 27-63, p. 42.

45. Michel Lussault, Hyper-lieux, Les nouvelles géographies de la mondialisation, Paris, Seuil, 2017, p. 249.

46. Olivier Razac, La grande santé, Paris, Flammarion, p. 202.

47. Robert Castel, La gestion des risques, Paris, Editions de Minuit, 1981, p. 136.

48. Michaël Foessel, « Interminable démocratie », in Esprit, octobre 2020, pp. 69-79.

49. Naomi Klein, "How big tech plans to profit from the pandemic », in The Guardian, 13 mai 2020, https://www.theguardian.com/news/2020/may/13/naomi-klein-how-big-tech-plans-toprofit-from-coronavirus-pandemic. Cet article a été traduit en partie dans le Courrier international: https://www.courrierinternational.com/long-format/long-format-naomi-kleinne-laissons-pas-les-geants-du-web-prendre-le-controle-de-nos

50. Grégoire Chamayou, Les corps vils, op. cit., p. 339.

51. Barbara Stiegler, "Il faut s'adapter », Sur un nouvel impératif politique, Paris, Gallimard, 2019, pp. 303-304.

\section{RÉSUMÉS}

Cet article entend approfondir une des hypothèses qui est au cœur de l'argumentaire du présent Cahier du GRM, à savoir que l'expérimentation constitue un concept clé pour comprendre la conjoncture de la crise sanitaire ou plutôt celle de la gestion de la crise. Il développe l'idée que, si le rapport savoir - pouvoir qui a sous-tendu la crise peut être qualifié de technocratique, le réel pouvoir de l'expertise réside moins dans l'imposition d'une vérité, que dans le pouvoir d'expérimenter. Après avoir illustré cette hypothèse d'une dynamique d'expérimentalisation 
généralisée, l'article en examine les effets sur un triple plan (subjectivation, temporalisation et spatialisation).

This article intends to explore one of the hypotheses that is at the heart of the argument of this Cahier $d u$ GRM, namely that experimentation is a key concept for understanding the health crisis or rather the management of the crisis. He develops the idea that, if the knowledge-power relationship that underpinned the crisis can be described as technocratic, the real power of expertise lies less in the imposition of a truth than in the power to experiment. After illustrating this hypothesis of a generalized experimentation dynamic, the article examines its effects on a triple level (subjectivation, temporalization and spatialization).

\section{INDEX}

Index géographique : Europe

Mots-clés : expertise, crise sanitaire, expérimentation, biopolitique

Keywords : expertise, health crisis, experimentation, biopolitics

\section{AUTEUR}

\section{ALAIN LOUTE}

Alain Loute est Professeur en éthique clinique à la Faculté de médecine et médecine dentaire de l'Université Catholique de Louvain et membre de l'Institut de recherche santé et société de cette même Université. Il a été Maître de conférences au Centre d'éthique médicale de l'Université Catholique de Lille et Chargé de recherches au Fonds National de la Recherche Scientifique au sein du Centre de Philosophie du Droit de l'UCLouvain. 